\title{
Cryptosporidium, chronic diarrhoea and the proximal small intestinal mucosa
}

\author{
A D Phillips, A G Thomas, J A Walker-Smith
}

\begin{abstract}
The association between Cryptosporidium, chronic diarrhoea and a proximal small intestinal mucosal enteropathy was reviewed over a six and a half year period. One hundred and twenty three children with cryptosporidiosis and no clinical evidence of immune deficiency were identified. $50 \%$ of children excreting only Cryptosporidium had chronic diarrhoea. Most cases $(63 \%)$ of chronic diarrhoea occurred in the first two years of life. A mild to moderate enteropathy was present in all nine children undergoing a small intestinal biopsy and seven showed the presence of Cryptosporidium adhering to villous epithelium. All patients eventually recovered spontaneously. Cryptosporidium is a cause of chronic diarrhoea and a proximal small intestinal mucosal enteropathy in children without immune deficiency. Screening for the parasite should be part of the investigative procedures in children with chronic diarrhoea.
\end{abstract}

(Gut 1992; 33: 1057-1061)

Electron Microsco

Department and
Academic Department of Paediatric

Gastroenterology, Queen Elizabeth Hospital for Children, London A D Phillips

A G Thomas

J A Walker-Smith

Correspondence to:

Dr A D Phillips, EM

Department, Queen Elizabeth

Hospital for Children

Hackney Road, Londo E2 8PS

Accepted for publication 28 November 1991

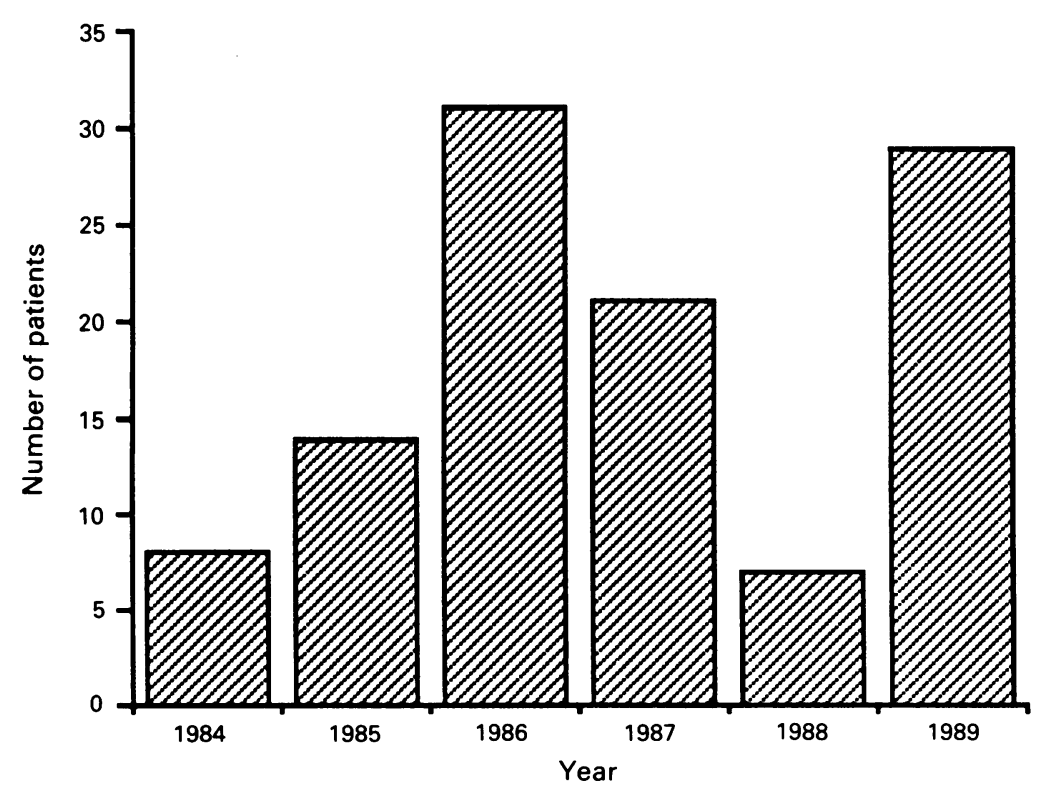

Figure 1: Cases of cryptosporidiosis per year. in $1907^{2}$ but the first human case was no recognised until $1976^{3}$ and only eight cases were described in the literature before $1982 .{ }^{4}$ Between 1982 and 1986 it changed from being described as an uncommon parasite ${ }^{4}$ to the most common parasite identified ${ }^{5}$ and over 11000 cases were reported between 1985 and $1988 . .^{6}$ There is good evidence that Cryptosporidium causes acute gastroenteritis as it has been found more frequently in the stools of children with diarrhoeal disease than controls $\mathrm{s}^{7-9}$ and has been detected in diarrhoeal outbreaks. ${ }^{10}{ }^{11}$ It has been accidently transferred from animals to $\operatorname{man}^{12}$ and has been experimentally transmitted from man to calves and mice. ${ }^{10}$ Cryptosporidium has been detected in the proximal small intestine, ${ }^{7}$ the large intestine and the rectum in man. ${ }^{313}$ Seroconversion has been shown ${ }^{14}$ but there are no human volunteer studies. Cases have been reported from Europe, Africa, India, the West Indies, North and South America indicating world wide prevalence.

Two main forms of clinical presentation have been described; a chronic, life threatening illness in the immunocompromised and an acute self limiting gastroenteritis in immunocompetent patients. ${ }^{15}$ Indeed, chronic diarrhoea has been an infrequently reported finding in studies of immunocompètent children with Cryptosporidium. We have previously reported two cases associated with chronic diarrhoea, failure to thrive and a proximal small intestinal enteropathy. ${ }^{16}$ In order to further study the relationship with chronic diarrhoea we have reviewed patients presenting at Queen Elizabeth Hospital for Children with cryptosporidiosis since 1983 when routine screening of the stools was introduced.

\section{Methods}

\section{PATIENTS}

Patients presenting at Queen Elizabeth Hospital for Children between October 1983 and April 1990 found to be excreting Cryptosporidium in the stool were reviewed retrospectively to assess the presenting symptoms, duration of diarrhoea and patient characteristics. Chronic diarrhoea was defined as diarrhoea persisting for at least 14 days. The routine microbiological examination of the stools included: negative staining electron microscopy for gastrointestinal viruses and light microscopy for ova, cysts, and parasites. Bacterial culture was performed in order to identify pathogens including Salmonella, enteropathogenic $E$ coli, Aeromonas, Campylobacter, and Shigella. Cryptosporidial oocysts were identified by a modified Ziehl-Nielsen stain as described previously.? The total number of patients who had stool samples analysed could only be accurately assessed in the one year period (1989) when records were computerised.

In nine patients with chronic diarrhoea and severe failure to thrive a proximal small intestinal biopsy was done using a pediatric twin port 


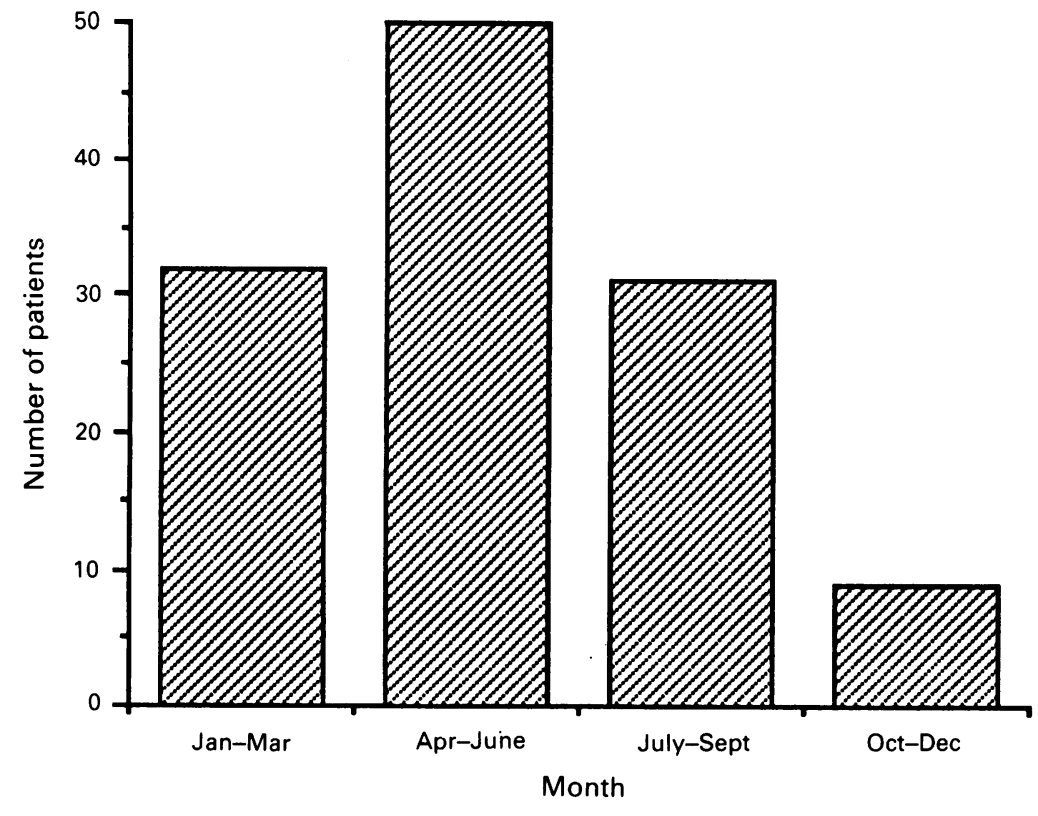

Figure 2: Accumulated cases of cryptosporidiosis per three month period.

biopsy capsule ${ }^{17}$ after obtaining informed consent. The small intestinal mucosa was studied by histology, morphometry,,$^{18}$ biochemistry, ${ }^{19}$ and electron microscopy. ${ }^{20}$ The immune competence of patients with the most severe symptoms was screened by studying serum immunoglobulins, lymphocyte transformation in response to phytohaemagglutinin stimulation, the formation of $T$ cell rosettes with sheep erythrocytes, yeast opsonisation, and neutrophil chemotaxis and nitro blue tetrazolium chloride reduction.

\section{Results}

In the 6.5 year study period 123 children had Cryptosporidium identified in the stool and the notes of 109 of these were found and reviewed. In the single one year period (1989) when computer records were available a total of 1662 children provided stools for microbiological examination and $27(1 \cdot 6 \%)$ had cryptosporidiosis. The annual figures (Fig 1) showed marked variation from
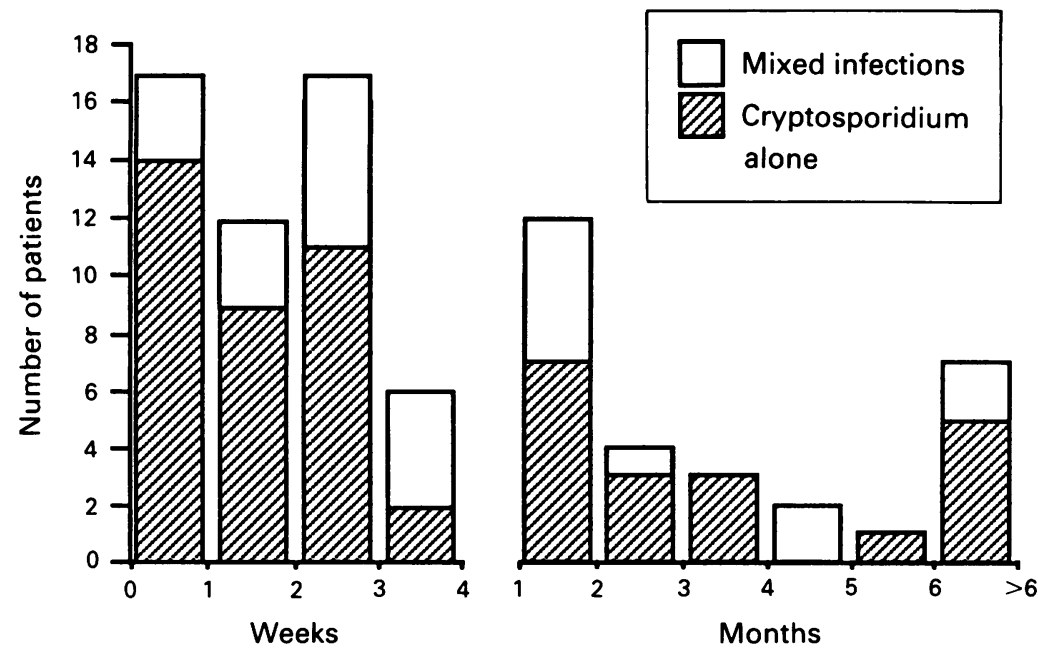

Duration of diarrhoea

Figure 3: Duration of diarrhoea in patients with cryptosporidiosis. year to year: on average 18 cases were identified per year, with fewer cases in 1984 and 1988, and more cases in 1986 and 1989. Significantly more cases of cryptosporidiosis occurred between April and June and less cases were seen between October and December (Fig 2) over the whole period of the study $\left(\chi^{2}\right.$ test, both $\left.\mathrm{p}<0.005\right)$.

The most common presenting symptom was diarrhoea in 100/109 (92\%) patients. This was usually watery and offensive with occasional mucus or blood staining. Fifty six patients $(51 \%)$ had vomiting and $23(21 \%)$ had significant abdominal pain. Nine patients without diarrhoea had unexplained pyrexia (three), nausea (one), abdominal pain (four), and/or vomiting (four). There was no clinical evidence of immunodeficiency in any of the 109 patients. Duration of diarrhoea was documented in 90/109 cases and is shown in Figure 3. Fifty two of 90 children (58\%) had chronic diarrhoea. In 35 cases (39\%) the diarrhoea persisted for at least 21 days and in seven cases $(8 \%)$ for more than six months. In all cases the diarrhoea eventually resolved spontaneously.

Sixty four of 90 children had Cryptosporidium alone, 32 of these $(50 \%)$ had diarrhoea persisting for 14 days or more, and 21 (33\%) had diarrhoea persisting for over 21 days. Half of the cases (32/ 64) occurred in children under two years of age and $20(63 \%)$ of these had chronic diarrhoea compared with only $38 \%$ (12/32) of children aged greater than two years $(\mathrm{p}<0.05)$. Details concerning the length of excretion of Cryptosporidium in relation to symptoms is not available in the majority of cases. Chronic diarrhoea was more frequent in those with mixed infections $(20 / 26(77 \%))$ compared with those with Cryptosporidium alone $(\mathrm{p}<0.025) ; 14$ had diarrhoea for 21 days or more. The other organisms detected in the children with mixed infections included Giardia lamblia, Aeromonas, enteropathogenic $E$ coli, Shigella, Salmonella, Campylobacter, rotavirus, adenovirus, calicivirus, and astrovirus. Nineteen children had one other organism, five children had two, and two children had three.

Weight was documented in $82 / 90$ and 19 (23\%) were below the third percentile. This was not related to the duration of diarrhoea $\left(\chi^{2}\right.$ test, $p>0.05)$. Seven children who were above the third percentile had recently lost weight or were failing to thrive. Although a greater proportion of patients with mixed infections had weight below the third percentile (eight of 21) this was not significantly different to those with Cryptosporidium alone (11/61).

Two patient subgroups were identified: these were children who had recently travelled abroad (18), and children who had an itinerant existence within the United Kingdom, generally living in caravan sites (17). Mixed infections were common in both (seven cases in the former subgroup, and six in the latter). Considering the children who were only excreting Cryptosporidium, chronic diarrhoea occurred in nine of 11 children $(82 \%)$ who had returned from travel abroad (Indian subcontinent (eight), Europe (two), and Africa (one)) and in seven of 11 children (64\%) from itinerant families (eight of nine and four of seven respectively having diarrhoea for greater than 21 days). In comparison 

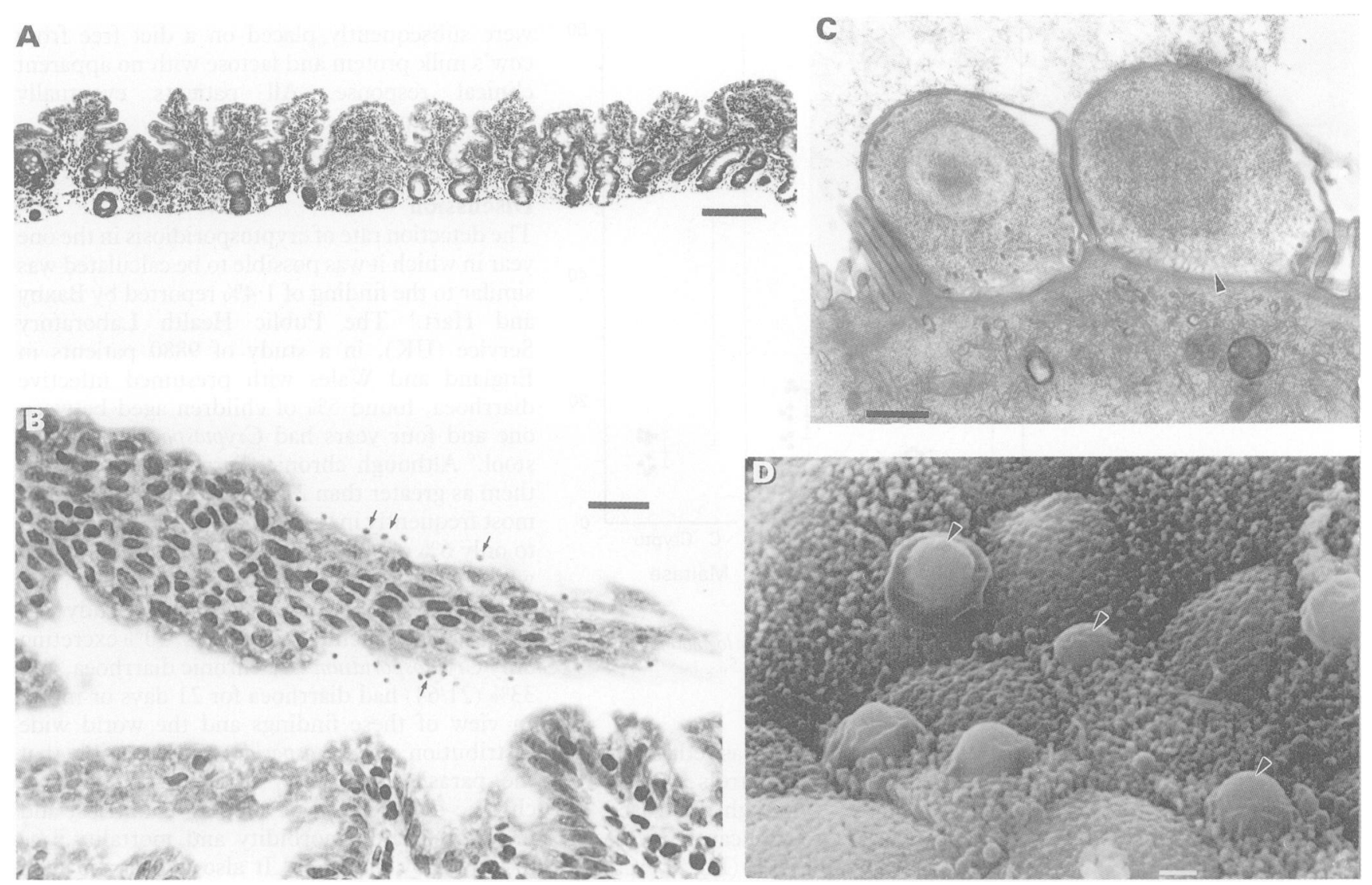

Figure 4: (a) Light microscopical appearance of small intestinal mucosa $($ Bar $=200 \mu \mathrm{m})$. (b) Light microscopy of Cryptosporidium adhering to upper villous surface (arrows) $($ Bar $=20 \mu \mathrm{m})$. (c) Transmission electron micrograph demonstrating Cryptosporidium adhering to the brush border with loss of microvilli and formation of attachment organelle (arrow) (Bar=500 nm). (d) Scanning electron microscopy showing Cryptosporidium adhering to the mucosal surface (arrows) $($ Bar $=1 \mu \mathrm{m})$.

Figure 5: Measurement of villous height and crypt depth. Abbreviations: $V H=$ villous height, $C D=$ crypt depth, $C=$ controls, Crypto=patients with cryptosporidiosis.

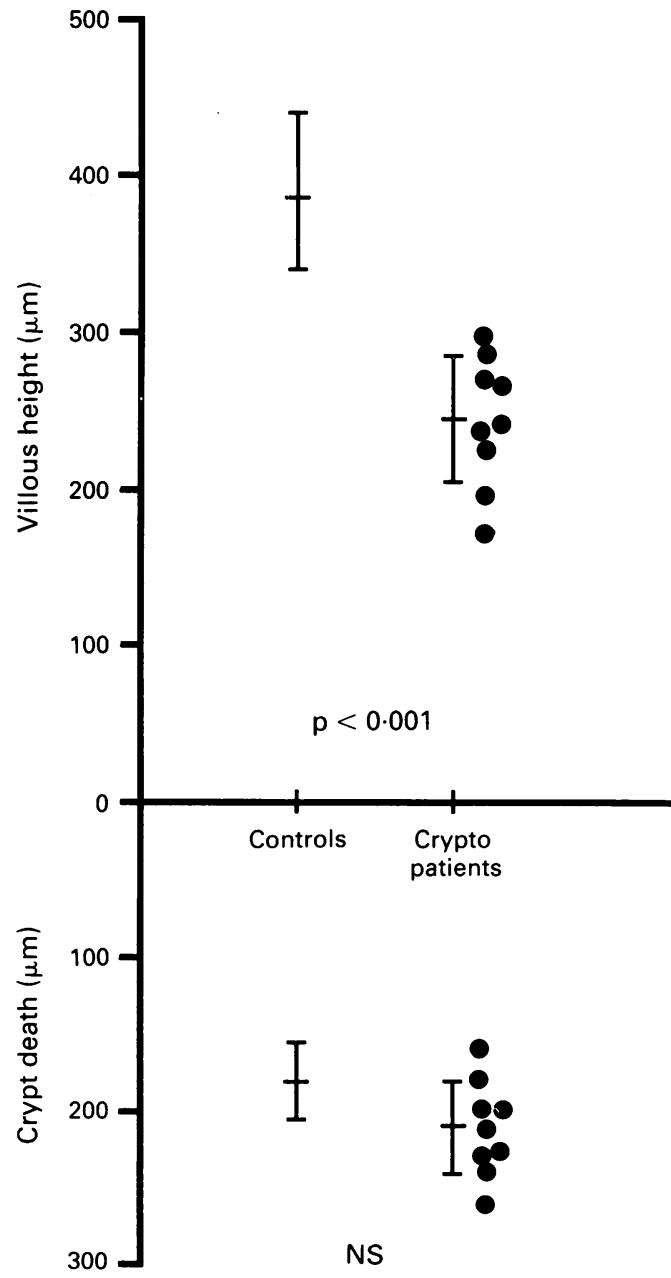

four of seven with the remaining cases, chronic diarrhoea was more frequent in those who had travelled abroad ( $\chi^{2}$ test, $\left.p<0.005\right)$, and was just significantly increased in patients from itinerant families $(\mathrm{p}<0.05)$.

Nine patients with Cryptosporidium, chronic diarrhoea and severe failure to thrive had a proximal small intestinal biopsy performed. The documented length of excretion of Cryptosporidium in these children was $60,20,19$, $15,8,7,7,1$, and 1 days. This information is incomplete however, as stool excretion was not regularly monitored and the latter two children only had single stools examined. One child had previous failure to thrive and developed diarrhoea which persisted for 14 days; the other eight failed to thrive after the onset of diarrhoea. In all eight cases the diarrhoea continued for over 21 days, in five it persisted for more than three months. Linear growth was impaired in four of the nine children.

Light microscopy of the small intestinal mucosa showed a mild to moderate enteropathy in all nine patients (Fig 4a). In seven of the nine patients Cryptosporidium was identified adhering to the mucosa. Cryptosporidium was found on villous, not crypt, epithelium (Fig 4b) and there was evidence of brush border effacement at sites of attachment (Fig 4c). Scanning electron microscopy, which affords a high resolution view of the mucosal surface, proved a very effective way of detecting the organism (Fig 4d). Giardia lamblia was identified in the mucosa in one patient but only Cryptosporidium was seen in the 


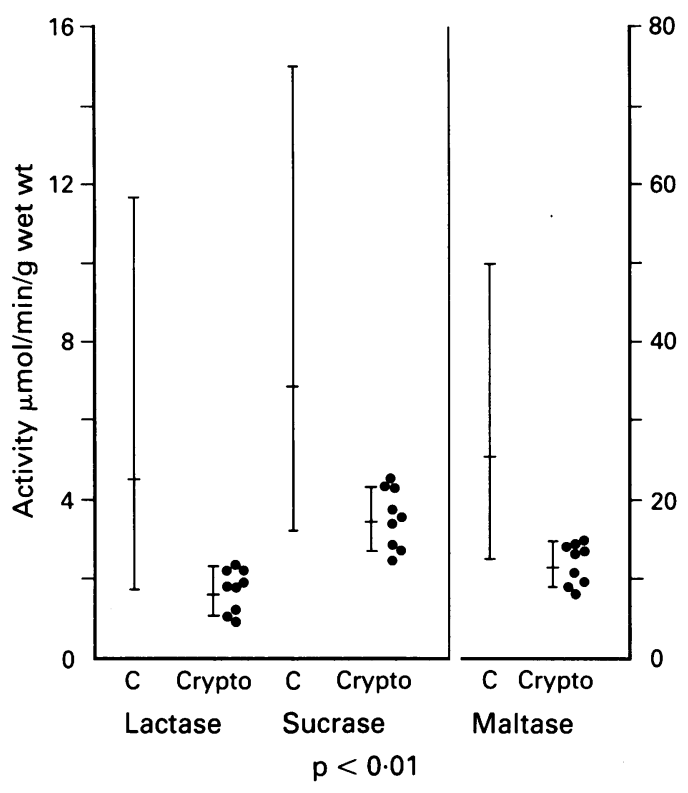

Figure 6: Disaccharidase activities. Note log normal distribution. Abbreviations as for Figure 5.

other patients. Villous height was reduced in all patients compared with controls $(p<0.001)$ whereas crypt depth, although increased in individual cases, was not significantly increased in the group as a whole ( $p>0 \cdot 05)$ (Fig 5). Activity of the disaccharidases lactase, sucrase, and maltase was significantly reduced compared with controls $(\mathrm{p}<0.01)$ (Fig 6) and the intraepithelial lymphocyte count was significantly increased $(\mathrm{p}<0.001)$ (Fig 7).

Immune function was screened in five of the nine patients requiring biopsy and all tests were normal.

Patients with diarrhoea were given oral rehydration therapy for 24 hours and then returned to their normal feeds. Five of the nine patients undergoing a small intestinal biopsy

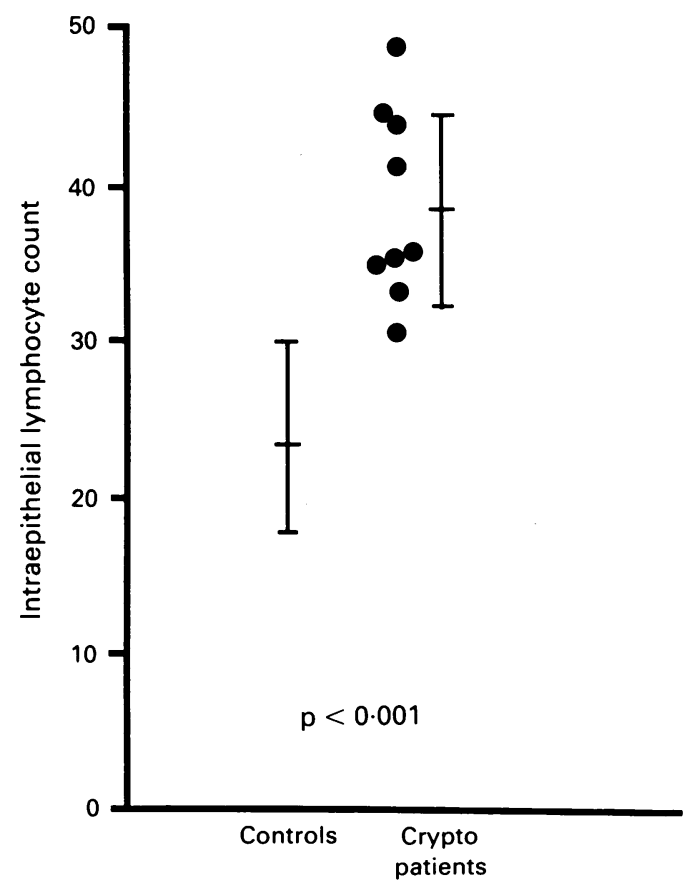

Figure 7. Intraepithelial lymphocyte count (IEL). Other abbreviations as for Figure 5. were subsequently placed on a diet free from cow's milk protein and lactose with no apparent clinical response. All patients eventually recovered spontaneously.

\section{Discussion}

The detection rate of cryptosporidiosis in the one year in which it was possible to be calculated was similar to the finding of $1.4 \%$ reported by Baxby and Hart. ${ }^{8}$ The Public Health Laboratory Service (UK), in a study of 9880 patients in England and Wales with presumed infective diarrhoea, found $5 \%$ of children aged between one and four years had Cryptosporidium in the stool. ${ }^{6}$ Although chronic diarrhoea (defined by them as greater than 21 days duration) occurred most frequently in this age group, this amounted to only $6 \%$ of cases - that is, $94 \%$ had diarrhoea which lasted for less than 21 days.

A surprising finding in the present study was that 32 of the 64 children - that is, $50 \%$ excreting only Cryptosporidium had chronic diarrhoea, and $33 \%(21 / 64)$ had diarrhoea for 21 days or more. In view of these findings and the world wide distribution of Cryptosporidium it is possible that the parasite may play an important role in chronic diarrhoea in developing countries, and in its associated morbidity and mortality,,$^{21}$ as indicated by others. ${ }^{22}{ }^{23}$ It also appears to be a cause of traveller's diarrhoea in early childhood, as reported in older children and adults, ${ }^{2+}$ and to be associated with chronic diarrhoea in such circumstances. Indeed, the reason for the greater frequency of chronic diarrhoea in this study in comparison with others in the United Kingdom, ${ }^{625}$ may be partly explained by the subgroups of children who had recently travelled abroad and those who had an itinerant existance. This highlights the remaining $50 \%(16 / 32)$ of cases with chronic diarrhoea for whom there is no ready explanation for the persistence of their symptoms. It is possible that the natural history of cryptosporidiosis in the United Kingdom includes diarrhoea extending up to 21 days. ${ }^{625}$ Nine of these 16 remaining children, however, had diarrhoea for over 21 days, indicating that host factors may be important in the continuation of their symptoms. Twenty nine per cent of cases had a mixed infection, and chronic diarrhoea was more frequent in these patients. There was insufficient evidence to determine if the mixed infections were concurrent, intercurrent, or sequential. Indeed, more information is required concerning the pattern of excretion of Cryptosporidium and other organisms, in order to investigate whether the chronic diarrhoea in these cases is a result of persistent infection or is a postinfective phenomenon. In general, in immunocompetent children, it appears that Cryptosporidium and Giardia lamblia ${ }^{26}$ infections may be associated with chronic diarrhoea, bacterial infections may extend into the chronic period,,$^{27}$ and viral infections are of short term duration. ${ }^{27} 28$

Around one quarter of the children in this study were below the third percentile for weight suggesting an association between cryptosporidiosis and failure to thrive, as described in other centres. 22325 In developing countries it is 
uncertain whether cryptosporidiosis leads to failure to thrive or whether malnutrition predisposes to cryptosporidiosis. ${ }^{22} 23$ These two postulates are not necessarily mutually exclusive. In this study infection with Cryptosporidium appears to be the primary event. Four of the nine patients requiring a small intestinal biopsy had impaired linear growth emphasising the potential serious effects of this illness. This also highlights the need to find an effective treatment, particularly as diagnosis is now relatively straightforward.

The association between a proximal small intestinal enteropathy and cryptosporidiosis in man has not been well established. In this study there was a remarkably consistent proximal small intestinal enteropathy of mild to moderate severity. Such an abnormality might be considered to be insufficient to explain the severity and chronicity of symptoms, however, involvement of widespread areas of the intestine including the colon have been described in cryptosporidiosis ${ }^{313}$ and the extent of the enteropathy may be an important factor in these cases.

The organism was only seen on villous epithelium. In immune deficient states Cryptosporidium is also found attached to crypt epithelium within the crypt lumen, ${ }^{29} 30$ indicating differences in the extent of colonisation of the mucosal surface in the two conditions. This observation is of interest in relation to the involvement of the immune system in influencing the topography of mucosal colonisation, and requires further investigation.

We conclude that Cryptosporidium is an important cause of chronic diarrhoea with failure to thrive in immunocompetent children and is associated with a proximal small intestinal enteropathy. Screening for Cryptosporidium should be routinely carried out in such children.

Part of these data were published as a letter (BMF 1990; 300: 1272-3).

1 Casemore DP. Epidemiological aspects of human cryptosporidiosis. Epidemiol Infect 1990; 104: 1-28.

2 Tyzzer EE. A sporozoan found in the peptic glands of the common mouse. Proc Soc Exp Biol Med 1907; 5: 12-3.

3 Nime FA, Burek JD, Page DL, Hoescher MA, Yardley JH. Acute enterocolitis in a human being infected with the protozoan Cryptosporidium. Gastroenterology 1976; the proto $70: 592-8$.

$4 \mathrm{Ma} \mathrm{P}$. Cryptosporidium and the enteropathy of immune deficiency. F Pediatr Gastroenterol Nutr 1984; 3: 488-90.

5 Holley HP, Dover C. Cryptosporidium: a common cause of parasitic diarrhoea in otherwise healthy individuals. F Infect 1986; 153: 365-8.
6 Public Health Laboratory Service Study Group. Cryptosporidiosis. BMF 1990; 300: 774-7.

7 Isaacs D, Hunt GH, Phillips AD, Price EH, Raafat F, WalkerSmith JA. Cryptosporidiosis in immunocompetent children. Smith JA. Cryptosporidiosis in

8 Baxby D, Hart CA. The incidence of cryptosporidiosis: a two year prospective study in a children's hospital. $\mathcal{F}$ Hyg Camb 1986; 96: 107-11.

9 Tzipori S, Smith M, Birch C, et al. Cryptosporidiosis in hospital patients with diarrhoea. Am $\mathcal{F}$ Trop Med Hyg 1983 32: $931-4$

10 Current WL, Reese NC, Ernst JV, Bailey WS, Heyman MB Weinstein WM. Human cryptosporidiosis in immunocompetent and immunodeficient persons. Studies of an outbreak and experimental transmission. $N$ Engl $\mathcal{F} \mathrm{Med}$ 1983; 308: 1252-7.

11 Alpert G, Bell LM, Kirkpatrick CE, Budnick LD, Campos JM, Friedman HM, Plotkin SA. Outbreak of cryptosporidiosis in a day-care centre. Pediatrics $1986 ; 77: 152-7$.

12 Blagburn BL, Current WL. Accidental infection of a researcher with human Cryptosporidium. F Infect Dis 1983; researcher

13 Fletcher A, Sims TA, Talbot IC. Cryptosporidial enteritis without general or selective immune deficiency. BMF 1982; 285: 22-3

14 Casemore DP. The antibody response to Cryptosporidium development of a serological test and its use in a study of immunologically normal persons. F Infect 1987; 14: 125-34.

15 Anonymous. Cryptosporidiosis. Lancet 1984; i: 492-3.

16 Phillips AD, Hunt G, Price E, Isaacs D, Raafat F, Larcher V, et al. Cryptosporidium in the postenteritis syndrome. In: Walker-Smith JA, McNeish AS, eds. Diarrhoea and malwutrition in childhood. Butterworths, London: 1984: 82-4.

17 Kutrition in childhood. Butterworths, Londiatric small intestinal biopsy capsule with two ports. Gut 1976; 17: 158-9

18 Maluenda C, Phillips AD, Briddon A, Walker-Smith JA. Quantitative analysis of small intestinal mucosa in cow's milk sensitive enteropathy. $\mathcal{F}$ Pediatr Gastroenterol Nutr 1984; 3: 349-56.

19 Phillips AD, Avigad S, Sacks J, Rice SJ, France NE, WalkerSmith JA. Microvillous surface area in secondary disaccharidase deficiency. Gut 1980; 21: 44-8.

20 Phillips AD, France NE, Walker-Smith JA. The structure of the enterocyte in relation to its position on the villus: an

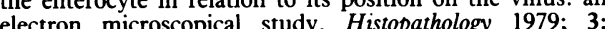
117-30.

21 Bhan MK, Arora NK, Ghai OP, Ramachandran K, Koshoo V, Bhadrai N. Major factors in diarrhoea related mortality among rural children. Indian $\mathcal{F} M$ Med Res 1986; 83: 9-12.

22 McFarlane DE, Horner-Bryce J. Cryptosporidiosis in well nourished and malnourished children. Acta Paediatr Scand 1987; 76: 474-7.

23 Sallon S, Deckelbaum RJ, Schmid II, Harlap S, Baras M, Spira DT. Cryptosporidium, malnutrition, and chronic diarrhoea in children. Am 7 Dis Child 1988; 142: 312-5.

24 Jokippi L, Pohjola S, Jokipii AMM. Cryptosporidiosis associated with travelling and giardiasis. Gastroenterology $1985 ; 89$. $838-42$.

25 Thomson MA, Benson JWT, Wright PA. Two year study of Cryptosporidium infection. Arch Dis Child 1987; 62: 559-63.

26 Welsh JD, Poley JR, Hensley J, Bhatia M. Intestinal disaccharidase and alkaline phosphatase activity in giardiasis. saccharidase and alkaline phosphatase activity

27 Ellis ME, Watson B, Mandal BK, Dunbar EM, Craske J, Curry A, et al. Micro-organisms in gastroenteritis. Arch Dis Child 1984; 59: 848-55.

28 Uhnoo I, Olding-Stenkvist E, Kreuger A. Clinical features of acute gastroenteritis associated with rotavirus, enteric adenoviruses, and bacteria. Arch Dis Child 1986; 61: 732-8.

29 Booth CC, Slavin G, Dourmashkin R, Donlach I, Webste D, Bird RG, et al. Immunodeficiency and cryptosporidiosis. BMF 1980; 281: 1123-7.

30 Kocoshis SA, Cibull ML, Davis TE, Hinton JT, Seip M, Banwell JG. Intestinal and pulmonary cryptosporidiosis in an infant with severe combined immunodeficiency. $\mathcal{F}$ Pediat Gastroenterol Nutr 1984; 3: 149-57. 